\title{
Cryptococcal Polysaccharides Induce L-Selectin Shedding and Tumor Necrosis Factor Receptor Loss from the Surface of Human Neutrophils
}

\author{
Zhao Ming Dong and Juneann W. Murphy \\ Department of Microbiology and Immunology, University of Oklahoma Health Science Center, Oklahoma City, Oklahoma 73190
}

\begin{abstract}
High titers of cryptococcal polysaccharides in the serum and spinal fluid and the lack of cellular infiltrates in the infected tissues are hallmarks of disseminated cryptococcosis. Cryptococcal polysaccharides given intravenously to mice inhibit the influx of leukocytes into sites injected with inflammatory mediators. The purpose of this investigation was to determine if cryptococcal polysaccharides, i.e., glucuronoxylomannan (GXM), galactoxylomannan, and mannoprotein, affect expression of molecules on the surface of neutrophils that are important in extravasation. GXM in the absence of serum was shown to induce human neutrophils to shed L-selectin, a molecule needed in the first step of neutrophil movement into tissues. In the presence of serum, GXM caused a further shedding of L-selectin. Shedding of L-selectin was evident by reduced amounts of L-selectin on the neutrophils treated with GXM and by increased levels of soluble L-selectin in the GXM-treated neutrophil supernatants. GXM also stimulated neutrophils to have reduced expression of TNF receptor. In contrast, GXM-treated neutrophils showed increased levels of CD15 and CD11b, and unchanged CD16 expression. In the absence of serum, galactoxylomannan and mannoprotein did not affect L-selectin, TNF receptor, CD15, CD11b, or CD16 on neutrophils but did induce loss of L-selectin in the presence of serum. Our results indicate that cryptococcal polysaccharides, especially GXM, can cause shedding of L-selectin from the surface of neutrophils, and this may prevent neutrophils from attaching to the endothelial cell surfaces. Blockage of this early step in cell migration from the vessels into tissues may be responsible in part for reduced cellular infiltration into infected tissues of individuals with disseminated cryptococcosis. (J. Clin. Invest. 1996. 97:689-698.) Key words: Cryptococcus neoformans • fungi • inflammation • adhesion molecules $\bullet$ Lewis $^{\mathrm{x}}$ carbohydrate
\end{abstract}

\section{Introduction}

Cryptococcus neoformans is an encapsulated yeast-like organism that causes infection in both immunologically normal and

Address correspondence to Dr. Juneann W. Murphy, Department of Microbiology and Immunology, University of Oklahoma Health Science Center, PO Box 26901, Oklahoma City, OK 73190. Phone: 405271-3110; FAX: 405-271-3117; E-mail: juneann-murphy@uokhsc.edu. Dr. Z.M. Dong's present address is The Center for Blood Research, Harvard Medical School, 800 Huntington Ave., Boston, MA 02115.

Received for publication 14 August 1995 and accepted in revised form 13 November 1995.

J. Clin. Invest.

(c) The American Society for Clinical Investigation, Inc.

0021-9738/96/02/0689/10 \$2.00

Volume 97, Number 2, February 1996, 689-698 immunocompromised individuals. Cryptococcosis ranks fourth in the infectious diseases causing death of AIDS patients $(1,2)$. Systemic cryptococcosis is characterized by high titers of cryptococcal polysaccharides in serum and minimal cellular infiltration into infected tissues of patients (3-5). By using a mouse model, we have found that an intravenous injection of cryptococcal polysaccharides inhibits leukocyte infiltration, including PMN infiltration, into the site of a $C$. neoformans-induced inflammatory response as well as into an inflammatory site induced by another organism, Mycobacterium tuberculosis, or induced by a proinflammatory cytokine such as TNF- $\alpha$ (6). Our findings suggest that the minimal cellular infiltrates observed in infected tissues of cryptococcosis patients may be due, in part, to the circulating cryptococcal polysaccharides. The fact that high titers of cryptococcal polysaccharides in serum of cryptococcosis patients can diminish leukocyte migration in response to stimuli other than $C$. neoformans may be an important confounding factor in AIDS patients with cryptococcosis.

The mechanisms underlying inhibitory effects of circulating cryptococcal polysaccharides on leukocyte influx are not clear. It is well known that leukocyte attachment to endothelial cells is an important step for leukocyte extravasation. PMN-endothelial cell interactions are mediated by two different sets of receptor-ligand interactions $(7,8)$. One set of receptors involves the selectin family and their ligands, and the other involves the $\beta 2$ integrins (CD11a,b,c/CD18) and their ligands. It is possible that circulating cryptococcal polysaccharides may downregulate PMN surface expression of functionally important selectins or integrin molecules and/or may block the receptorligand interactions by binding to the receptor or the ligand. Any of these interactions of cryptococcal polysaccharides would result in inhibition of the PMN attachment to the endothelial cells, which in turn would inhibit PMN migration into inflammatory sites.

L-selectin is one adhesion molecule constitutively expressed on the surface of most leukocytes including human neutrophils (PMN), and it is essential for the first contact between endothelial cells and $\mathrm{PMN}$ as the PMN are recruited into an inflammatory site (9). Anti-L-selectin mAb and L-selectinbinding polysaccharide, fucoidin, given intravenously, have been found to block leukocyte accumulation within the inflamed peritoneum and spinal fluid of bacterial meningitis patients $(10,11)$, showing that L-selectin is involved in the inflammatory response. Down-regulation of L-selectin on leukocytes is a well-known phenomenon (9). Proteolytic cleavage of the extracellular region of L-selectin results in formation of soluble L-selectin (sL-selectin), ${ }^{1}$ which has been proposed to

1. Abbreviations used in this paper: CMI, cell mediated immune; CneF, culture filtrate of Cryptococcus neoformans; GalXM, galactoxylomannan; GXM, glucuronoxylomannan; MP, mannoprotein; PE, phycoerythrin; PHS, pooled human serum; sL-selectin, soluble L-selectin; TNFR, TNF receptor. 
have regulatory functions (12). Loss of L-selectin from the PMN surface before adhesion of the PMN to the endothelial cells is one mechanism by which intravascular IL-8 induces blockage of PMN accumulation at the site of inflammation (13).

Filtrate from a 5-d culture of C. neoformans (CneF) contains major cryptococcal capsular polysaccharides (14). CneF can be fractionated into at least three components (15). The predominant component in $\mathrm{CneF}$ is a high-molecular weight glucuronoxylomannan (GXM). GXM is readily detected in serum from patients with systemic cryptococcosis by polyclonal anticryptococcal antibodies on latex beads. The two other minor constituents in $\mathrm{CneF}$ are galactoxylomannan (GalXM) and mannoprotein (MP). Indirect evidence suggests that GalXM and MP are also shed into the patient's body fluids during infection (16). In our previous studies, we have found that an intravenous injection of each component of $\mathrm{CneF}$ into mice can block PMN accumulation at the site of inflammation (6).

The main objective of this study was to determine whether or not individual components of CneF (GXM, GalXM, or MP) can directly and/or indirectly induce L-selectin shedding from the surface of human PMN in vitro. To assess this, expression of L-selectin on human PMN after incubation with and without individual components of CneF in HBSS or in pooled human serum (PHS) was measured by immunofluorescence staining of cells and flow cytometric analysis. sL-selectin levels in supernatants of PMN stimulated with individual components of CneF were determined with a commercial ELISA kit. To determine if only L-selectin was affected by cryptococcal polysaccharides, the densities of other PMN surface molecules, such as TNF receptor (TNFR), CD16 (FcyRIII), CD15 (a Lewis $^{\mathrm{X}}$ carbohydrate antigen), and CD11b, were measured after treatment of PMN with HBSS or individual components of CneF.

\section{Methods}

Maintenance of endotoxin-free conditions. To prevent endotoxin from influencing the experimental results, all experiments were performed under conditions that minimize endotoxin contamination. This included the use of plastic ware that was endotoxin-free, glassware that had been heated for $3 \mathrm{~h}$ at $180^{\circ} \mathrm{C}$, and reagents that had been found to contain $<8 \mathrm{pg} / \mathrm{ml}$ of endotoxin (minimal detectable level) with the Limulus assay (Whittaker Bioproducts, Inc., Walkersville, MD).

$m A b s$. The following fluorochrome-conjugated mAbs were purchased from Becton Dickinson Immunocytometry Systems, Inc. (Mountain View, CA): FITC-conjugated anti-CD15 (mouse IgM), phycoerythrin (PE)-conjugated anti-CD16 (mouse IgG1), PE-conjugated anti-L-selectin mAb anti-Leu-8 (mouse IgG2a), PE-conjugated anti-CD11b (mouse IgG2a), and PE-conjugated mouse irrelevant $\mathrm{IgG} 1$ and $\mathrm{IgG} 2 \mathrm{a}$ for isotype controls. FITC-conjugated mouse irrelevant IgM for an isotype control was purchased from Coulter Immunology (Hialeah, FL). Rat anti-human TNFR p75-80 (IgG2b) was purchased from Genzyme (Cambridge, MA). FITC-conjugated goat $\mathrm{F}\left(\mathrm{ab}^{\prime}\right) 2$ anti-rat IgG, used as a secondary reagent to recognize antiTNFR p75-80, and rat irrelevant IgG2b, used as an isotype control for anti-TNFR p75-80, were purchased from Caltag Laboratories (South San Francisco, CA). Anti-GXM mAb 439 (mouse IgG1) was a generous gift of Dr. T. R. Kozel (University of Nevada, Reno, Nevada). The mouse myeloma protein MOPC21, used as an isotype control for the mouse IgG1 antibodies, was obtained from Organon TeknikaCappel (Malvern, PA).

Preparation of GXM, GalXM, and MP from CneF. CneF was prepared as previously reported by culturing C. neoformans isolate $184 \mathrm{~A}$ in a defined medium (17). CneF was analyzed by anodic polyacrylamide slab gel electrophoresis and displayed three bands previously characterized as GXM, GalXM, and MP after staining the gels with periodic acid-Schiff's stain (14). GXM, GalXM, and MP fractions of CneF were prepared as previously described (18). Briefly, GXM was isolated from $\mathrm{CneF}$ by gel filtration on a high-resolution column (Sephacryl S-300; Pharmacia Biotech, Uppsala, Sweden). The GalXM fraction was separated from the MP fraction by concanavalin A chromatography. All fractions were subjected to electrophoresis and concanavalin A blotting to determine the efficiency of the fractionation and were then lyophilized and reconstituted in endotoxinfree, sterile physiologic saline solution to $2 \mathrm{mg}$ (dry wt) $/ \mathrm{ml}$.

Immunoprecipitated GXM. GXM at $1 \mathrm{mg}$ dry $\mathrm{wt} / \mathrm{ml}$ was incubated with anti-GXM $(500 \mu \mathrm{g} / \mathrm{ml})$ or as a control with an irrelevant isotype-matched $\mathrm{mAb}(500 \mu \mathrm{g} / \mathrm{ml})$ for $30 \mathrm{~min}$ at $4^{\circ} \mathrm{C}$. Then protein G-Sepharose was added, and the mixture was incubated for an additional $30 \mathrm{~min}$ at $4^{\circ} \mathrm{C}$. After centrifugation, the supernatants were collected as the source of anti-GXM-treated GXM or isotype-matched antibody-treated GXM.

PHS and complement C5-deficient human serum. Peripheral blood was collected from three volunteers. The sera were pooled and stored at $-130^{\circ} \mathrm{C}$. This pool was used as the source of PHS. Human serum deficient in the complement component C5 was purchased from Sigma Chemical Co. (St. Louis, MO).

Human neutrophils. Human PMN were obtained by the method previously described (18). Briefly, buffy coats of human peripheral blood were diluted with HBSS and layered on Ficoll-Hypaque and centrifuged. The cell pellet was collected from the Ficoll-Hypaque and sedimented with $6 \%$ dextran solution for $30 \mathrm{~min}$. The PMN-rich population was collected from the upper layer of the dextran solution. Residual red blood cells were lysed with a $0.85 \% \mathrm{NH}_{4} \mathrm{Cl}$ solution. PMN were washed and resuspended in HBSS before they were diluted to a concentration of $1 \times 10^{7}$ cells $/ \mathrm{ml}$. The cell populations were comprised of $>95 \%$ neutrophils as determined by differential counts on Diff-Quick (Baxter Healthcare Co., Miami, FL)-stained smears. The viability of cells in the PMN-enriched fraction was $>95 \%$ by trypan blue dye exclusion.

Cell cultures. PMN $\left(10^{6}\right)$ were incubated in $0.5-\mathrm{ml}$ polypropylene microcentrifuge tubes for the designated time at $37^{\circ} \mathrm{C}$ either in $100 \mu \mathrm{l}$ HBSS alone or in $100 \mu \mathrm{l}$ HBSS containing FMLP at $10^{-6} \mathrm{M} ; 50 \mu \mathrm{g}$ or $100 \mu \mathrm{g}$ of GXM, GalXM, or MP; $25 \%$ PHS or C5-deficient serum with or without added GXM, GalXM, or MP; anti-GXM-treated GXM; or isotype-matched antibody-treated GXM. Solutions of PHS or C5-deficient serum with added GXM, GalXM, or MP were incubated in a $37^{\circ} \mathrm{C}$ water bath for 30 min before they were mixed with PMN. After culture of PMN for the designated time (30 min, $1 \mathrm{~h}$, or $2 \mathrm{~h}$ ) with one of the reagents, the supernatant fluid was tested for the presence of sL-selectin with an L-selectin ELISA kit, and PMN were immunostained for surface expression of L-selectin, TNFR p75-80, $\mathrm{CD} 16, \mathrm{CD} 15$, or CD11b and subjected to flow cytometric analysis. The viability of each PMN population was determined by trypan blue dye exclusion after incubation with each one of the above reagents for $2 \mathrm{~h}$ at $37^{\circ} \mathrm{C}$ and found to be the same as when the PMN were incubated similarly in HBSS ( $>90 \%$ viable).

Immunofluorescence staining and flow cytometric analysis. For direct immunofluorescent analysis of L-selectin, CD15, CD11b, or $\mathrm{CD} 16$ on the surface of human PMN, $10^{6} \mathrm{PMN}$ were suspended in 50 $\mu \mathrm{l}$ of staining buffer (PBS supplemented with $0.1 \%$ BSA and $0.1 \%$ sodium azide) containing $0.2 \mu \mathrm{g}$ of PE-conjugated mouse anti-Leu- 8 $\mathrm{mAb}, 2 \mu \mathrm{g}$ of FITC-conjugated mouse anti-CD15 mAb, $0.5 \mu \mathrm{g}$ of PEconjugated mouse anti-CD11b mAb, or $1 \mu \mathrm{g}$ of PE-conjugated mouse anti-CD16 $\mathrm{mAb}$ and incubated for $30 \mathrm{~min}$ at $4^{\circ} \mathrm{C}$. After three washes with staining buffer, PMN were fixed in $1 \%$ paraformaldehyde (wt/ vol) in PBS and stored at $4^{\circ} \mathrm{C}$ in the dark until analyzed. All samples were analyzed within $24 \mathrm{~h}$ after labeling. Isotype-matched FITC or PE-conjugated $\mathrm{mAbs}$ that did not specifically react with human PMN were used as controls to exclude Fc-related and nonspecific binding. For indirect immunofluorescent analysis of the TNFR p75-80 on the 
surface of human PMN, $10^{6} \mathrm{PMN}$ were suspended in $50 \mu$ l of staining buffer with $1 \mu \mathrm{g}$ goat anti-rat IgG (Sigma Chemical Co.) as a blocking antibody and incubated for $30 \mathrm{~min}$ at $4^{\circ} \mathrm{C}$. After washing twice in staining buffer, the cells were suspended in $50 \mu \mathrm{l}$ of staining buffer containing $0.5 \mu \mathrm{g}$ of rat anti-human TNFR p75-80 (IgG2b) mAb or the isotype-matched control antibody and incubated for $30 \mathrm{~min}$ at $4^{\circ} \mathrm{C}$. After three washes, the cells were suspended in staining buffer containing $1 \mu \mathrm{g}$ of FITC-conjugated goat $\mathrm{F}\left(\mathrm{ab}^{\prime}\right) 2$ anti-rat IgG and incubated for $30 \mathrm{~min}$ at $4^{\circ} \mathrm{C}$. After three more washes in staining buffer, the cells were fixed and stored as indicated above.

The immunofluorescently labeled cells were analyzed with a FACStar ${ }^{\circledR}$ PLUS flow cytometer (Becton Dickinson Immunocytometry Systems, Inc.). Single-color immunofluorescence analysis was performed on all samples, and the fluorescent label on the isotype control antibody was the same as the fluorescent label on the specific antibody. FITC and PE were excited using the 488-nM line from an argon ion laser (Coherent Inc., Palo Alto, CA) set at $100 \mathrm{~mW}$. FITC fluorescence was detected through a 530/30 band pass filter, and PE fluorescence was detected through a 575/26 band pass filter. Neutrophils were identified for analysis by forward and right-angle light scatter, and gates were set to exclude from the analyses contaminating dead cells, erythrocytes, lymphocytes, platelets, or debris. Routinely, 10,000 cells were analyzed. The data were displayed as histograms with the $\mathrm{x}$-axis showing the fluorescence intensity and the $y$-axis showing the relative number of cells. To determine the percent positive cells, a channel marker was set on the appropriate control histogram so that $5 \%$ or less of the cells were to the right of the channel marker, and then the percentage of cells that appeared to the right of the channel marker in the specific antibody-stained sample histogram was determined. The mean log fluorescence intensity (channel number) for the total PMN population and for the positive cells was determined and converted into linear fluorescence intensity units. The results (mean fluorescence intensity units) are equivalent to net fluorescence intensity units (experimental sample fluorescence units minus control sample fluorescence units).

$s L$-Selectin ELISA. Quantification of sL-selectin levels in supernatants of PMN stimulated with FMLP, GXM, GalXM, or MP were performed as described in the kit insert with a commercial ELISA kit (Bender MedSystems, Vienna, Austria). This commercial assay was capable of detecting from 0.4 to $25 \mathrm{ng} / \mathrm{ml}$ of sL-selectin.

Statistical analysis. Mean, SEM, and Student's $t$ test results were used to analyze the data. When two groups were compared, a $P$ of $\leq 0.05$ was considered to indicate a significant difference between the groups.

\section{Results}

Effects of GXM, GalXM, and MP on surface L-selectin expression. It has been reported that L-selectin is constitutively expressed on the surface of blood PMN from normal individuals
(19). With a direct immunofluorescence labeling procedure and PE-conjugated anti-Leu- $8 \mathrm{mAb}$, we found that the majority (80-90\%) of human peripheral blood PMN expressed L-selectin (Table I). It is well documented that PMN shed their surface L-selectin very rapidly (within $5 \mathrm{~min}$ ) after stimulation with FMLP and do not reexpress this molecule (9). In preliminary experiments, we confirmed that culturing PMN with FMLP for 30 min resulted in a dramatic decrease in the amount of cell surface L-selectin, and, with further incubation up to $1 \mathrm{~h}$, we did not observe any further changes in L-selectin on the PMN surfaces. As shown in Table I, $~ 60 \%$ of the PMN were L-selectin negative within $1 \mathrm{~h}$ after stimulation with FMLP, and the remaining $40 \%$ of the PMN expressed $\sim 50 \%$ of the level of L-selectin expressed on unstimulated PMN. The density of L-selectin on the total PMN population after stimulation with FMLP was reduced $76 \%$. GXM at $100 \mu \mathrm{g} / 10^{6}$ PMN, like FMLP, induced loss of L-selectin from human PMN. Throughout our studies, we examined FMLP- and GXM-induced L-selectin loss with PMN from 10 different donors, and, although the degree of L-selectin loss varied from individual to individual, in each case both stimulants induced a loss in L-selectin from the surface of the PMN. In general, L-selectin loss induced by GXM was less than that induced by FMLP (Table I); however, in some individuals, GXM induced an equivalent or greater loss than FMLP. Our preliminary experiments, in which L-selectin loss was measured at 30 and 60 min after addition of the stimulant, demonstrated that the GXM-induced loss of L-selectin from PMN was slower than the FMLP-induced loss inasmuch as maximal L-selectin loss did not occur until $1 \mathrm{~h}$ after addition of GXM, whereas maximal loss of L-selectin in response to FMLP was evident at $30 \mathrm{~min}$. In contrast to GXM, neither GalXM nor MP in HBSS induced L-selectin loss from human PMN after incubation for $1 \mathrm{~h}$.

In previous studies, we found that both GXM and MP could activate the complement pathway in serum to generate a chemotactic factor(s), possibly C5a (18). In those studies, it was not determined whether or not GalXM could activate the complement pathway. Other investigators have reported that C5a can induce L-selectin loss from PMN (20). Therefore, it is possible that the activation of complement by GXM, GalXM, or MP results in the production of a factor(s) as C5a that induces L-selectin loss. To examine this possibility, GXM, GalXM, or MP was incubated with $25 \%$ PHS for $30 \mathrm{~min}$ at $37^{\circ} \mathrm{C}$ before the activated serum was incubated with PMN. As shown in Fig. 1, PHS alone did not affect L-selectin density on the PMN. GXM induced L-selectin loss in the absence of se-

Table I. L-Selectin Expression on Human PMN after Incubation with Various Reagents*

\begin{tabular}{|c|c|c|c|}
\hline Reagent ${ }^{\ddagger}$ & $\begin{array}{l}\text { Percent PMN positive } \\
\quad \text { for L-selectin }\end{array}$ & $\begin{array}{l}\text { Mean fluorescence intensity units } \\
\text { of L-selectin-positive PMN }\end{array}$ & $\begin{array}{l}\text { Mean fluorescence intensity } \\
\text { units of total PMN population }\end{array}$ \\
\hline HBSS & $86 \pm 5$ & $52 \pm 9$ & $38 \pm 4$ \\
\hline FMLP & $41 \pm 13^{\S}$ & $19 \pm 4^{\S}$ & $9 \pm 2^{\S}$ \\
\hline GXM & $58 \pm 7^{\S}$ & $26 \pm 8^{\S}$ & $12 \pm 1^{\S}$ \\
\hline GalXM & $88 \pm 4$ & $53 \pm 9$ & $41 \pm 7$ \\
\hline MP & $87 \pm 4$ & $52 \pm 10$ & $39 \pm 4$ \\
\hline
\end{tabular}

*L-Selectin on PMN was determined by flow cytometric analysis after immunostaining of treated PMN with PE-conjugated anti-L-selectin mAb. Mean fluorescence intensity units were calculated as described in Methods. The results are reported as mean \pm SEM from four normal donors. ${ }^{\ddagger}$ PMN $\left(10^{6}\right)$ were incubated with HBSS with FMLP at $10^{-6} \mathrm{M}$, or with $100 \mu \mathrm{g}$ of GXM, GalXM, or MP in $0.1 \mathrm{ml}$ of $\mathrm{HBSS}$ for $1 \mathrm{~h}$ at $37^{\circ} \mathrm{C}$. ${ }^{\S} \mathrm{P}<0.01 \mathrm{vs} \mathrm{HBSS}$ controls. 


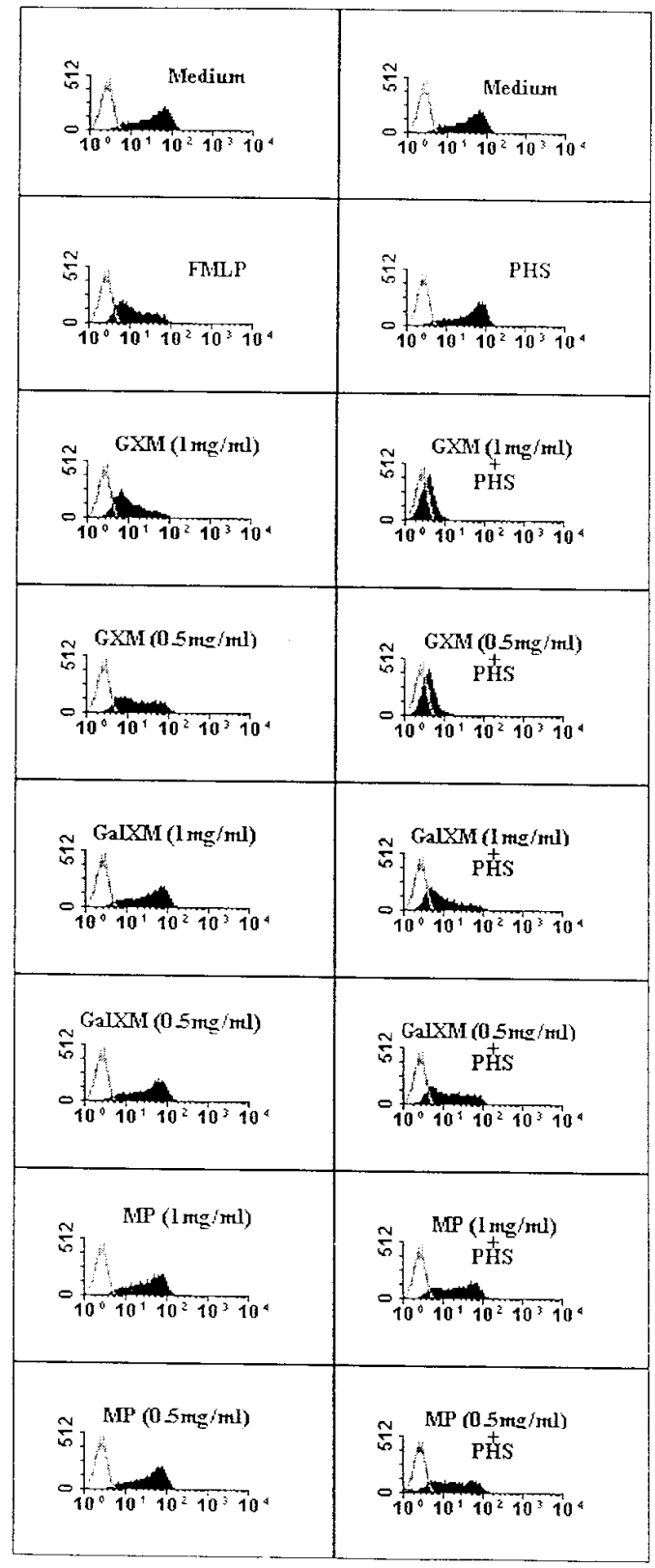

Figure 1. Flow cytometric analysis of L-selectin expression on human PMN after incubation with GXM, GalXM, or MP in HBSS medium or in PHS at $37^{\circ} \mathrm{C}$ for $1 \mathrm{~h}$. (y-axis) Relative number of cells; ( $x$-axis) $\log$ fluorescence intensity. Shaded areas represent anti-L-selectin (PE-conjugated anti-Leu-8)-positive cells; unshaded areas represent cells labeled with a PE-conjugated IgG2a irrelevant control mAb. $\mathrm{GXM}, \mathrm{GalXM}$, or MP in $25 \% \mathrm{PHS}$ was incubated in a $37^{\circ} \mathrm{C}$ water bath for $30 \mathrm{~min}$ before it was mixed with PMN. Data are from one representative experiment. The experiment was repeated two additional times with similar results.

rum, and, when mixed with serum, GXM induced additional L-selectin loss $(P<0.01$ when compared with incubation with GXM in HBSS). Neither GalXM nor MP induced L-selectin loss in the absence of serum, but, in the presence of serum, both GalXM and MP induced significant losses of L-selectin from PMN $(P<0.01)$. To examine which serum factor(s) might be important in the GXM-, GalXM-, or MP-activated serum that mediates L-selectin loss from PMN, serum defi-
Table II. L-Selectin Expression on Human PMN after Incubation with GXM, GalXM or MP in HBSS, PHS or C5-Deficient serum*

\begin{tabular}{|c|c|c|c|c|}
\hline & \multirow[t]{2}{*}{ Reagent } & \multicolumn{3}{|c|}{$\begin{array}{l}\text { Mean fluorescence intensity units of the total } \\
\text { PMN population after incubation with GXM, } \\
\text { GalXM, or MP suspended in: }\end{array}$} \\
\hline & & HBSS & PHS & C5-deficient PHS \\
\hline \multirow{4}{*}{ Experiment 1} & HBSS & 30 & 31 & 32 \\
\hline & GXM & 11 & 4.5 & 9 \\
\hline & GalXM & 28 & 6 & 33 \\
\hline & MP & 31 & 9 & 24 \\
\hline \multirow{4}{*}{ Experiment 2} & HBSS & 34 & 33 & 32 \\
\hline & GXM & 12 & 4 & 11 \\
\hline & GalXM & 33 & 10 & 31 \\
\hline & MP & 33 & 14 & 32 \\
\hline
\end{tabular}

*L-Selectin on PMN was determined after PMN $\left(10^{6}\right)$ were incubated with HBSS or with $100 \mu \mathrm{g}$ of GXM, GalXM, or MP in $0.1 \mathrm{ml}$ HBSS, PHS, or C5-deficient serum for $1 \mathrm{~h}$. After treatment, the PMN were labeled with PE-conjugated anti-L-selectin $\mathrm{mAb}$ and subjected to flow cytometric analysis. Mean fluorescence intensity units were calculated as described in Methods.

cient in the complement component C5 was treated with GXM, GalXM, or MP before adding the activated C5-deficient serum to PMN. As shown in Table II, in the presence of C5-deficient serum, neither GalXM nor MP induced L-selectin loss from the surface of PMN, and GXM-activated C5-deficient serum did not induce any greater L-selectin loss from PMN than was observed when PMN were incubated with GXM in HBSS. These data confirm that only GXM can directly induce L-selectin loss from PMN, and GXM, GalXM, and MP have similar abilities to activate the complement pathway that results in production of a C5-dependent serum factor(s) capable of causing L-selectin loss from PMN.

Effects of anti-GXM mAb on GXM-induced L-selectin loss. To demonstrate that GXM but not some potential contaminant(s) was directly inducing L-selectin loss from the surface of PMN, experiments were done with an anti-GXM mAb. In an initial experiment, we found that, when PMN were mixed with anti-GXM mAb and GXM for $1 \mathrm{~h}$, there was loss of L-selectin from the PMN surface. In subsequent experiments, we found that the L-selectin loss could be induced by anti-GXM mAb (IgG1) alone at $0.5 \mathrm{mg} \mathrm{mAb} / \mathrm{ml}$. Recently, other investigators have reported that stimulation of PMN with an irrelevant (anti-insulin) $\mathrm{mAb}$ resulted in loss of L-selectin from PMN, and the L-selectin loss was triggered by the mAb binding to Fc receptors, especially CD16 on the PMN (21). To avoid anti-GXM mAb-inducing L-selectin loss, which would interfere with our blocking test, the GXM preparation was incubated with anti-GXM or the isotype-matched $\mathrm{mAb}$ for 30 min at $4^{\circ} \mathrm{C}$ before removing immune complexes and free $\mathrm{IgG}$ with protein $\mathrm{G}-$ Sepharose. The efficiency of this procedure was confirmed with acrylamide gel electrophoresis, showing that there was no detectable GXM or IgG after treating the GXM preparation with anti-GXM mAb and protein G-Sepharose. After treatment with the supernatant from the immunoprecipitated GXM, the PMN were assessed for surface L-selectin. Removal of GXM by pretreatment with anti-GXM mAb eliminated the ability of the GXM preparation to induce L-selectin 


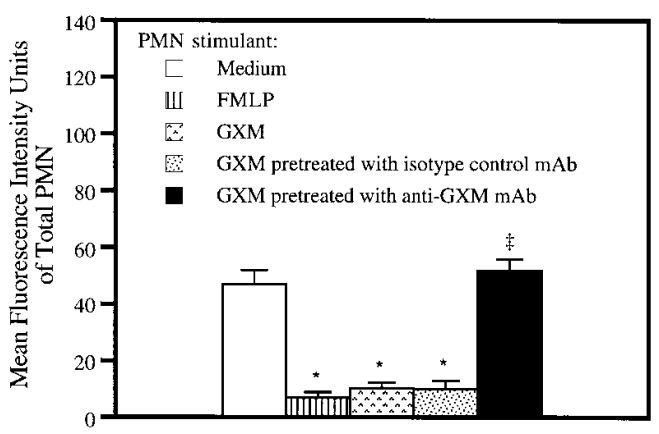

Figure 2. Effects of anti-GXM mAb on GXM-induced L-selectin loss from human PMN. GXM was incubated with IgG1 isotype control $\mathrm{mAb}$ or anti-GXM mAb, and then the immunoprecipitate and $\mathrm{IgG}$ were removed. L-selectin on the PMN surface was determined after treatment of the PMN with the designated reagent. Mean fluorescence intensity units were calculated as described in Methods. Data are representative of two experiments. $* P<0.01$ compared with medium control; ${ }^{\ddagger} P>0.05$ compared with medium control.

loss from PMN (Fig. 2). In contrast, after treating GXM with isotype control $\mathrm{mAb}$ and protein $\mathrm{G}-$ Sepharose, there was still GXM present but no free IgG as determined by acrylamide gel electrophoresis, and the preparation retained its ability to induce L-selectin loss from the PMN surfaces (Fig. 2). These results provide further evidence that GXM can directly induce down-regulation of L-selectin expression on PMN.

Quantification of sL-selectin levels in supernatants of PMN stimulated with GXM, GalXM, or MP. Loss of L-selectin from the surface of PMN after incubation with GXM at $37^{\circ} \mathrm{C}$ for $1 \mathrm{~h}$ could be due to shedding of L-selectin from the surface of PMN or to internalization of L-selectin by PMN. The main purpose of the experiment shown in Fig. 3 was to determine which of these two possibilities was the cause of the observed reduction in L-selectin from the surface of PMN after subjection to GXM. With an sL-selectin ELISA kit, the medium from freshly isolated PMN cultured for $1 \mathrm{~h}$ at $37^{\circ} \mathrm{C}$ contained low but detectable concentrations of sL-selectin (Fig. 3), suggesting that cell surface proteolysis may be part of the normal

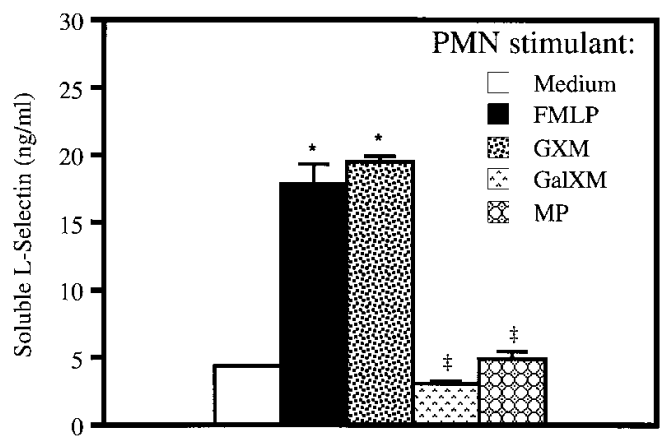

Figure 3. Quantification of sL-selectin levels in supernatants of PMN $\left(10^{6}\right)$ after incubation in $0.1 \mathrm{ml}$ of HBSS medium alone, $10^{-6} \mathrm{M}$ FMLP, or $100 \mu \mathrm{g}$ of GXM, GalXM, or MP at $37^{\circ} \mathrm{C}$ for $1 \mathrm{~h}$. sL-Selectin was measured in the PMN supernatants with a commercial ELISA. Values represent the mean \pm SEM obtained in quadruplicate determinations for each sample. Data are from one representative experiment of two. ${ }^{*} P<0.01$ compared with medium control; ${ }^{\ddagger} P>0.05$ compared with medium control. turnover of L-selectin. Stimulation of PMN with FMLP or GXM resulted in an increase in sL-selectin in the supernatants (Fig. 3). Incubation of PMN with GalXM or MP, which did not alter the cell surface L-selectin expression, did not cause an increase above control levels in sL-selectin in the supernatants (Fig. 3). The increase in sL-selectin in the supernatants from FMLP- or GXM-treated PMN was proportional to the potency of the stimulant to induce loss of L-selectin from the PMN surface. These data indicate that GXM stimulation of PMN, like FMLP stimulation, causes L-selectin to shed from the PMN surface.

Effects of GXM, GalXM, and MP on the surface TNFR p75-80, CD16, CD15, and CD11b expression. It is well known that expression of other types of membrane molecules such as TNFR (22) and CD16 (low affinity FcyR) (21, 23) are regulated by the enzymatic cleavage from the surface of cells, a mechanism similar to the down-regulation of L-selectin expression on PMN. To determine whether GXM induced only L-selectin loss, PMN treated with GXM were assessed for surface expression of TNFR and CD16, two receptors susceptible to enzymatic cleavage along with CD15, a Lewis X carbohydrate antigen, and CD11b that are not controlled by the enzymatic cleavage $(10,24)$.

There are two types of TNFR found on the surface of PMN: TNFR p55-60 and TNFR p75-80 (25). In this study, we only observed effects of GXM on PMN surface expression of TNFR p75-80 because anti-TNFR p55-60 mAb obtained from Genzyme did not stain the cells sufficiently for flow cytometric analysis. With the anti-TNFR p75-80 mAb in an indirect im-

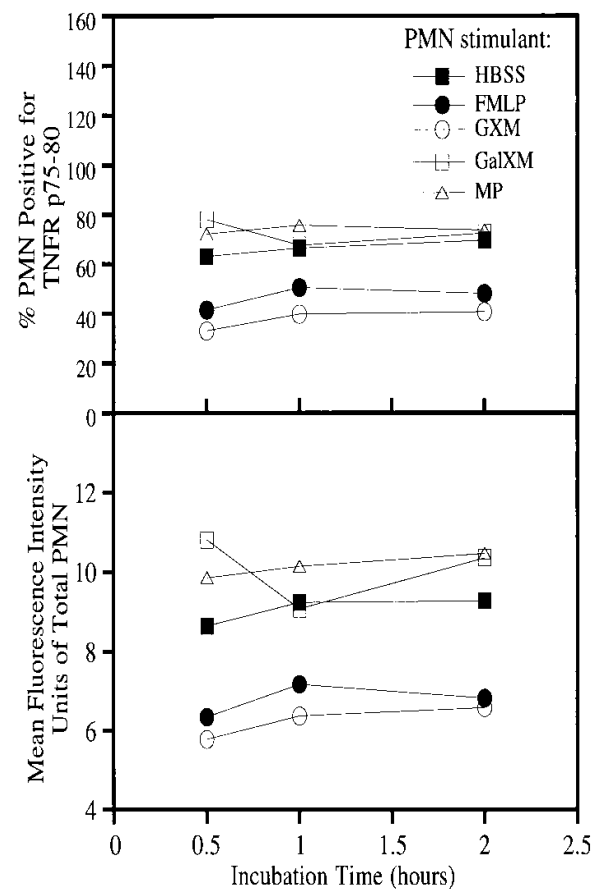

Figure 4. Effects of FMLP, GXM, GalXM, and MP on surface TNFR p75-80 expression on human PMN. PMN $\left(10^{6}\right)$ were incubated with $100 \mu \mathrm{g}$ of GXM, GalXM, or MP in $0.1 \mathrm{ml}$ of HBSS for $30 \mathrm{~min}, 1 \mathrm{~h}$, or $2 \mathrm{~h}$ at $37^{\circ} \mathrm{C}$. After treatment, the levels of TNFR p75-80 on the PMN were determined by flow cytometric analysis after indirect immunofluorescence staining. Mean fluorescence intensity units were calculated as described in Methods. Data are representative of three experiments using PMN obtained from three different donors. 


\section{$\mathrm{L}$-Selectin}

Medium

FMLP

GXM

GaIXM

MP
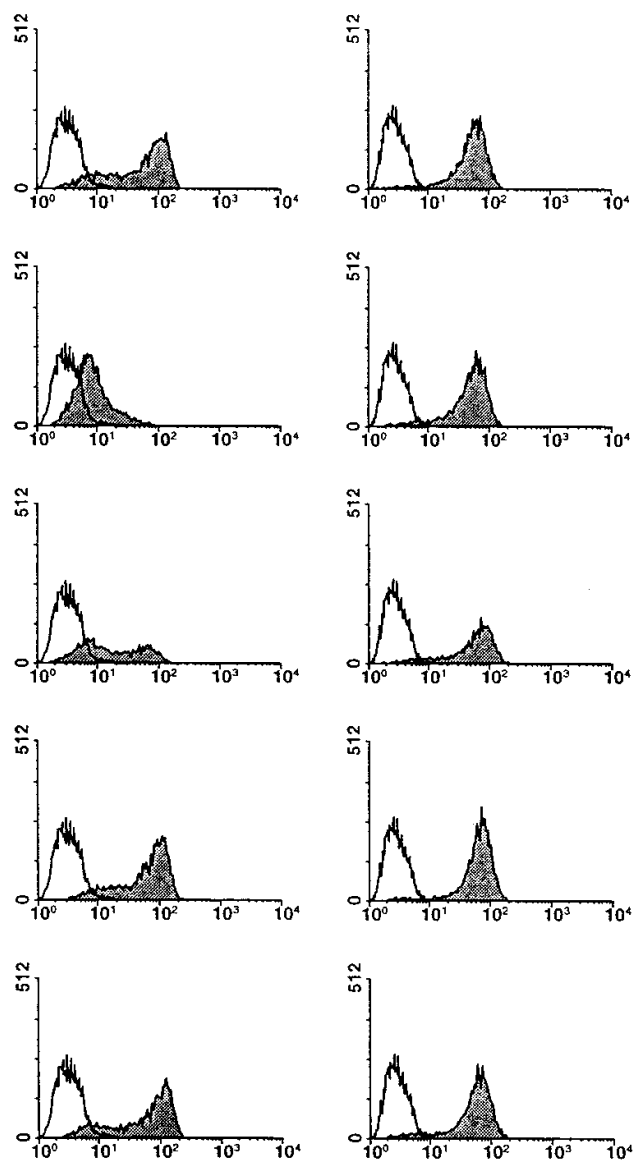

CD15
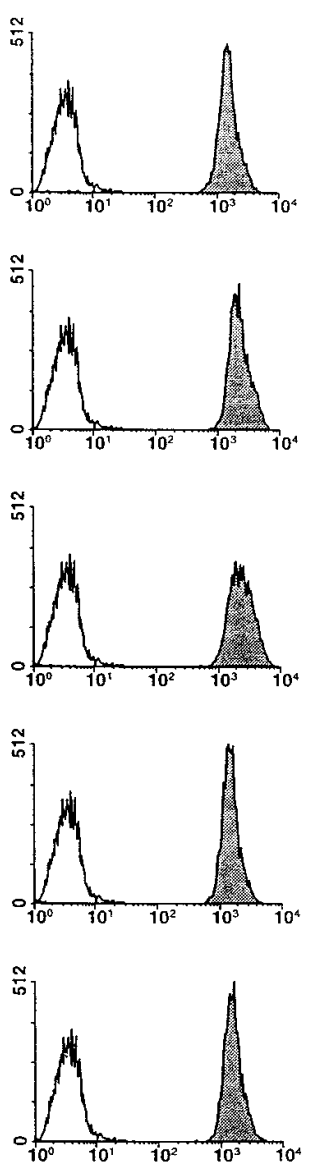

\section{Log Fluorescence}

Figure 5. Fluorescence intensity histograms showing effects of FMLP, GXM, GalXM, and MP on L-selectin, CD16, and CD15 expression on human PMN. Shaded areas represent PMN labeled with anti-L-selectin, anti-CD16, or anti-CD15 PMN; unshaded areas represent PMN labeled with the appropriate irrelevant isotype control. PMN $\left(10^{6}\right)$ were incubated with $100 \mu \mathrm{g}$ of GXM, GalXM, or MP in $0.1 \mathrm{ml}$ of HBSS for $1 \mathrm{~h}$ at $37^{\circ} \mathrm{C}$, and then L-selectin, CD16, or CD15 on the PMN was determined. The PMN were subjected to flow cytometric analysis after direct immunofluorescent staining of cells with PE-conjugated antiLeu-8 (L-selectin), PE-conjugated anti-CD16, or FITC-conjugated anti-CD15 mAb. Data are from one representative experiment of three. munofluorescence assay, we found that $60-75 \%$ of peripheral blood PMN from three normal donors expressed TNFR p7580 even after culturing the cells in HBSS for $30 \mathrm{~min}$ at $37^{\circ} \mathrm{C}$ (Fig. 4). Furthermore, the percentage of TNFR p75-80-positive cells was not significantly altered by extending the incubation time in HBSS to 1 or $2 \mathrm{~h}$ (Fig. 4). Both FMLP and GXM induced a significant loss of TNFR p75-80 from the surface of PMN $(P<0.05$ compared with PMN incubated in HBSS). Within 30 min of stimulation with FMLP, there were $35 \%$ fewer TNFR-positive cells than in controls that were incubated in HBSS, and the density of TNFR p75-80 on the total PMN population was reduced by $25 \%$ (Fig. $4, P<0.05$ ). GXM displayed greater activity in inducing TNFR p75-80 loss from the surface of PMN than did FMLP (Fig. 4). Within 30 min of stimulation with GXM, there were 50\% fewer TNFR p75-80positive cells than in the controls, and the density of TNFR p75-80 on the total PMN population was reduced by $35 \%$ (Fig. $4, P<0.01)$. The loss of TNFR p75-80 from PMN after incubation with GXM or FMLP was still evident after $2 \mathrm{~h}$ (Fig. 4). Neither GalXM nor MP induced loss of TNFR p75-80 from the surface of the PMN. In contrast, within 30 min of incubation with GalXM but not MP, the density of TNFR p75-80 on the surface of the total PMN population from all three tested individuals was higher ( $25 \%$ increase on average) than that on control PMN (those incubated in HBSS for $30 \mathrm{~min})(P<0.05)$
(Fig. 4). This increase appeared to be transient, in that the increase was not seen after a 1- or 2-h incubation (Fig. 4).

As shown in Fig. 5, we found that, after culturing PMN from three normal donors in HBSS medium at $37^{\circ} \mathrm{C}$ for $1 \mathrm{~h}$, $90 \%$ of the cells were CD16 positive. In the same experiments, both FMLP and GXM induced L-selectin loss from the surface of the PMN but did not affect CD16 expression.

PMN from three normal donors were $99 \%$ positive for CD15. Within $1 \mathrm{~h}$ of stimulation of PMN with FMLP or GXM, the expression of CD15 (mean fluorescence intensity units) was above control levels (Fig. 5 and Table III, $P<0.01$ ). After stimulation with FMLP, the density of CD15 on the total PMN population increased 60,24 , and $25 \%$ in the three different individuals, respectively. Similarly, after stimulation with GXM, the density of CD15 on the total PMN population increased 54,29 , and $48 \%$ in three individuals. As shown in Table III, in addition to up-regulation of CD15 expression, GXM also induced an increase in CD11b cell surface expression $(P<0.01)$. Neither GalXM nor MP altered CD15 or CD11b expression on the PMN surface.

\section{Discussion}

C. neoformans is the only encapsulated yeast-like organism that is pathogenic to humans. The capsular components of $C$. 
Table III. Effects of FMLP, GXM, GalXM, and MP on CD15 and CD11b Expression on Human PMN*

\begin{tabular}{lllc}
\hline & & \multicolumn{2}{c}{$\begin{array}{c}\text { Mean fluorescence intensity units } \\
\text { of the total PMN population } \\
\text { after incubation with the } \\
\text { designated reagent followed by } \\
\text { immunostaining with: }\end{array}$} \\
\cline { 3 - 4 } Reagent & Anti-CD15 & Anti-CD11b \\
\hline Experiment 1 & HBSS & 1485 & 656 \\
& FMLP & $1843(24)^{\ddagger}$ & $949(45)$ \\
& GXM & $1911(29)$ & $1203(83)$ \\
& GalXM & 1433 & 597 \\
& MP & 1596 & 592 \\
& HBSS & 1382 & 750 \\
& FMLP & $2207(60)$ & $1120(49)$ \\
& GXM & $2129(54)$ & $1405(87)$ \\
& GalXM & 1334 & 698 \\
& MP & 1485 & 698 \\
\hline
\end{tabular}

*PMN $\left(10^{6}\right)$ were incubated with FMLP at $10^{-6} \mathrm{M}$, or with $100 \mu \mathrm{g}$ of GXM, GalXM, or MP in $0.1 \mathrm{ml}$ of HBSS for $1 \mathrm{~h}$ at $37^{\circ} \mathrm{C}$. CD15 and CD11b expression were determined by immunofluorescence staining with FITC-conjugated anti-CD15, or PE-conjugated anti-CD11b and subjected the cells to flow cytometric analysis. Mean fluorescence intensity units were calculated as described in Methods. ${ }^{\ddagger}$ Values in parentheses indicate percent increase in the density of CD15 or CD11b on PMN after incubation with the designated reagent compared with incubation with HBSS medium.

neoformans are produced in great abundance in vivo and can be found in body fluids of patients with disseminated cryptococcosis $(3,15)$. Similar cryptococcal capsular components can be recovered from in vitro cultures $(14,15)$. The culture filtrate collected from in vitro growth medium of $C$. neoformans has been found to contain $88 \%$ GXM and $12 \%$ GalXM and MP together (15). It is known that $\sim 90-100 \%$ of the patients with disseminated cryptococcosis have measurable levels of cryptococcal polysaccharides, especially GXM, in their serum (26). Indirect evidence suggests that GalXM and MP are also found in sera of patients with cryptococcosis (16). In fact, detection of cryptococcal polysaccharides, especially GXM, in patients' sera by the latex agglutination assay is diagnostic for cryptococcosis. In non-AIDS patients, high or increasing serum titers of cryptococcal polysaccharide indicate progressive disease, whereas decreasing titers are accompanied by clinical improvement (3). Titers of cryptococcal polysaccharides in both cerebrospinal fluid and serum from AIDS patients with disseminated cryptococcosis are enormously elevated, frequently exceeding 1:100,000 (equivalent to $\sim 1 \mathrm{mg}$ cryptococcal polysaccharide/ml) and going as high as 1:2,000,000 (27). With therapy, the cryptococcal polysaccharide levels in cerebrospinal fluid from patients with AIDS generally decrease at a predictable rate; however, serum cryptococcal polysaccharide levels do not usually diminish dramatically (27). Cryptococcal polysaccharides have been shown to have several adverse effects on host defenses against $C$. neoformans. Cryptococcal polysaccharides are antiphagocytic (28), and they can induce suppression of both the humoral and cell-mediated immune (CMI) responses (29-31). Recently, we have found that an intravenous injection of cryptococcal polysaccharides into mice inhibits leukocyte accumulation at the site of a $C$. neoformans-induced inflammatory response (6), that is reminiscent of the limited cellular influx observed in infected tissues from patients with disseminated cryptococcosis (3-5). In the study presented here, we demonstrated yet another effect of cryptococcal polysaccharides, especially GXM, on host cells: GXM can remodel the surface of the PMN by inducing the loss of functionally important surface molecules such as L-selectin and TNFR. This latter phenomenon attributed to GXM may in fact serve as the mechanism by which PMN are prevented from accumulating in C. neoformans-infected tissues.

PMN are regarded as one of the effective components of the host's natural cellular defenses, and PMN can aid in early clearance of $C$. neoformans from the host $(32,33)$. Recently, Buchanan and Murphy found that PMN were one of the infiltrating cell types in the anticryptococcal CMI response (17), suggesting that PMN may participate in CMI-mediated late elimination of $C$. neoformans cells from the host. If the PMN act as effector cells against $C$. neoformans, irrespective of whether their action is early or later in the infection, PMN must first migrate to the site of infection. PMN migration from the vascular space into areas of inflammation is initiated by attachment of the PMN to specific receptors on the endothelial cell surfaces. According to a recent model, PMN-endothelial cell interactions are active processes requiring at least three steps $(7,8)$. First, the PMN-endothelial cell interactions are initiated by the binding of PMN surface molecules such as L-selectin to the endothelial cell surface counterreceptors. This initial adhesion is transient and causes rolling of the PMN along the endothelial cell surface. In the second step, the PMN are stimulated by specific chemoattractants or cell contactmediated signals that trigger activation or up-regulation of a second set of adhesion molecules such as the $\beta 2$ integrins and induce L-selectin shedding to stop rolling. Simultaneously, the ligands for the $\beta 2$ integrins are induced on the endothelial cell surface. Third, engagement of adhesion molecules such as $\beta 2$ integrins on PMN with their ligands such as ICAM-1 (CD54) on the endothelial cells will cause the PMN to stick firmly to the endothelial cells. After the three steps occur, PMN will migrate through the endothelial wall by yet undefined mechanisms. The PMN migration is under the direction of chemoattractants present at the inflammatory sites. The three initial steps in the migration process occur in sequence, not in parallel, because inhibition of any one of these steps gives essentially complete, rather than partial, inhibition of PMN emigration (8). A number of investigations have indicated that, if the initial L-selectin-mediated rolling is blocked, the sequential events involved in PMN migration into tissues will not occur and the inflammatory response will be abrogated $(10,11,13)$. Therefore, when cryptococcal polysaccharides, especially GXM, accumulate in the bloodstream, they will induce L-selectin loss from PMN before the first step of the PMN-endothelial cell interactions occurs. Consequently, circulating cryptococcal polysaccharides can block PMN infiltration into the site of inflammation by preventing L-selectin-mediated rolling.

GXM is considered to be a prominent virulence factor for C. neoformans (15). MP is the primary component recognized by the anticryptococcal CMI response in mice (14). The role of GalXM in pathogenicity is unknown. In our previous studies, it was found that intravenous injection of GalXM or MP also inhibited PMN accumulation at the sites of inflammation (6). In this respect, GalXM and MP are similar to GXM, suggest- 
ing that both GalXM and MP may also modulate PMN functions. The data presented here show that neither GalXM nor MP can directly induce loss of L-selectin and TNFR from the surface of PMN. So, in this respect, GalXM and MP contrast with GXM. It is well established that the surface of $C$. neoformans is a strong activator of the complement cascade (34), and both GXM and MP have been previously shown to have such activity (18). GalXM has not been assessed for its ability to activate the complement pathway. Since GalXM, like MP in the presence of PHS but not in the presence of C5-deficient serum, stimulates L-selectin loss from PMN, it is likely that GalXM also activates the complement cascade, resulting in production of C5a. C5a has been found by other investigators to induce L-selectin loss from the surface of PMN (20). Therefore, high levels of C5a in the intravascular space induced by high titers of cryptococcal polysaccharides (GXM, GalXM, or MP) in serum may contribute to the in vivo loss of L-selectin from the surface of circulating PMN and consequently to the inhibition of PMN migration into the infected tissues.

Down-regulation of L-selectin expression is a well-known phenomenon, and it is thought that down-regulation of L-selectin expression on PMN is due to accelerated proteolytic cleavage of L-selectin from the cell surface $(9,12)$. Adherence alone, without any other stimuli, also causes release of L-selectin from the PMN surface (12). This may explain why there are low but detectable levels of sL-selectin found in supernatants from 1-h cultures of PMN in HBSS medium. Both FMLP and GXM seem to accelerate this process, resulting in a greater amount of L-selectin shedding from the cell surface into the medium. High concentrations of sL-selectin have been found to inhibit L-selectin-dependent leukocyte attachment (12), indicating that sL-selectin induced by GXM may also play a role in GXM-mediated inhibition of PMN accumulation in tissues.

In addition to L-selectin, various other membrane molecules, including CD16 and TNFR, have been shown to be enzymatically cleaved from the surface of PMN $(22,23)$. Although the exact cell surface enzymes involved in the cleavage processes have not been defined, it has been suggested that there is no universal enzyme responsible for the cleavage of the different receptors expressed on a single cell population (21). The metalloprotease(s) and serine protease(s) have been found to be involved in the cleavage of CD16 but not in the cleavage of L-selectin on PMN (21); thus, one would expect cleavage of CD16 and L-selectin to be two independent modifications of the PMN surface. Consistent with the current concept, our data demonstrate that both FMLP and GXM induce L-selectin but not CD16 loss from the surface of PMN. Our findings suggest that both FMLP and GXM are able to activate an enzyme(s) for cleaving L-selectin through a pathway that does not affect the activity of the enzyme(s) for cleaving CD16. Both FMLP and GXM induce parallel loss of L-selectin and TNFR from the surface of PMN, suggesting that there may be a common mechanism or enzyme(s) responsible for cleaving L-selectin and TNFR.

TNF, a potent proinflammatory cytokine, plays a major role in inflammation and host defense (35). TNF exerts its effect on PMN, as well as on other inflammatory cells, by binding to two types of TNFR, the TNFR p55-60 and the TNFR p75-80 (25, 36). Both the p55-60 and p75-80 TNFR appear to be regulated by the same mechanisms. For example, adherence or stimulation of PMN with FMLP causes a parallel release of both TNFR p55-60 and TNFR p75-80 from the PMN
(37). Based on the fact that GXM induces TNFR p75-80 loss from PMN, we predict that TNFR p55-60 expression on PMN would be reduced after stimulation with GXM. Direct evidence for this could not be obtained because the anti-TNFR p55-60 available to us did not bind sufficiently to the PMN for use in flow cytometry. PMN with reduced TNFR would be expected to have diminished capabilities to respond to TNF at the site of an anticryptococcal CMI response. In contrast to findings with GXM stimulation, PMN after incubation with GalXM for 30 min appear to have higher numbers of surface TNFR than after incubation with HBSS medium. This could be due to GalXM blocking adherence-dependent down-regulation of TNFR expression on PMN or up-regulating TNFR expression on PMN, or both. Future studies are required to determine which of these possibilities is correct.

CD15 (Lewis ${ }^{\mathrm{X}}$ or fucosyl- $N$-acetyl-lactosamine), a carbohydrate antigen, is present on several PMN glycoproteins, including $\beta 2$ integrins such as CD11b/CD18 and the carcinoembryonic antigen-related molecule NCA-160 (CD66c) (38). In this study, we found that GXM up-regulated CD15 expression on PMN, suggesting that GXM may also up-regulate CD15-associated molecules such as CD11b/CD18 expression. Consistent with this suggestion, we observed that GXM also up-regulates CD11b expression on PMN. It is well documented that $\mathrm{CD} 11 \mathrm{~b} / \mathrm{CD} 18$ is stored in granules in PMN and up-regulation of $\mathrm{CD} 11 \mathrm{~b}$ expression is associated with degranulation of PMN $(39,40)$. Therefore, up-regulation of CD15 and CD11b expression on PMN by GXM indicates that the GXM activates the PMN.

Most chemoattractants such as FMLP and IL- 8 induce a concomitant down-regulation of L-selectin and up-regulation of CD11b expression on PMN and display both proinflammatory and antiinflammatory effects depending on their distribution between extravascular and intravascular spaces, respectively $(13,20,41,42)$. In previous studies, we have demonstrated that GXM is a chemoattractant both in vitro and in vivo for PMN $(6,18)$. For instance, in modified Boyden chemotactic chambers, GXM induces migration of human PMN across the membrane (18). In naive mice, injection of a GXM-enriched preparation such as $\mathrm{CneF}$ (which contains $88 \%$ GXM) into gelatin sponges implanted subcutaneously induces PMN infiltration into the sponges (6). In these cases, GXM acts as a chemoattractant and displays proinflammatory effects. As mentioned above, intravenous injection of CneF or GXM into mice inhibits PMN infiltration into sites of inflammation. Thus, intravascular GXM displays antiinflammatory effects (6). Therefore, when one considers that GXM is a chemoattractant and acts as other chemoattractants when in extravascular or intravascular spaces, the finding that GXM can induce down-regulation of L-selectin and up-regulation of CD11b expression on PMN is not surprising. Our results showing that GXM down-regulates L-selectin and up-regulates CD11b and CD15 on the surface of human PMN are similar to findings of other investigators who have shown that water-soluble membrane proteins from Helicobacter pylori, Escherichia coli, and Staphylococcus aureus induce L-selectin loss and increases in CD11b and CD15 expression on human PMN (43).

It has been reported that loss of L-selectin from the surface of PMN can be induced either by cellular activators (activation dependent) or by cross-linking of L-selectin with a chemical cross-linker or with specific mAbs (activation independent) (44). Although natural ligands on inflamed endothelial cells 
for L-selectin have not been identified (9), the presence of a lectin-like domain at the amino terminus of L-selectin strongly suggests that L-selectin may recognize a carbohydrate structure on the endothelial cell surface. The phosphomannan monoester, a soluble complex polysaccharide from yeast, and fucoidin, a homopolymer of sulfated L-fucose, are known to bind to L-selectin $(11,45)$. Therefore, it is possible that GXM might bind to L-selectin, resulting in cross-linking-mediated L-selectin loss. However, at present, we do not know whether GXMinduced L-selectin shedding from PMN is via an activationdependent mechanism or an activation-independent mechanism, or both. Our ongoing studies are addressing these questions.

In summary, we have shown that GXM, a high-molecular weight polysaccharide produced in abundance by pathogenic isolates of $C$. neoformans, acts similarly to typical chemoattractants such as FMLP and IL-8. GXM is chemotactic for human and mouse neutrophils $(6,18)$, it induces L-selectin shedding and the loss of TNFR from the PMN surface, and it stimulates the up-regulation of surface CD11b and CD15 on PMN. High concentrations of cryptococcal polysaccharides in serum of patients with cryptococcosis may exert these effects on peripheral blood PMN. The remodeling of the PMN surface through inducing loss of such functionally important molecules as L-selectin and TNFR may be responsible in part for cryptococcal polysaccharide-mediated antiinflammatory effects observed in patients with disseminated cryptococcosis.

\section{Acknowledgments}

The assistance of Michael Steele in assaying TNFR expression on PMN and help of Jim Henthorn and Joe Dynlacht in flow cytometric analysis are greatly appreciated. Flow cytometric analysis was performed at the Flow Cytometry and Cell Sorting Core Facility of Oklahoma Center for Molecular Medicine (Oklahoma City, OK).

This work was supported by Public Health Service grant AI18895 from the National Institute of Allergy and Infectious Diseases.

\section{References}

1. Armstrong, D. 1988. Life-threatening opportunistic fungal infection in patients with the acquired immunodeficiency syndrome. Ann. NY Acad. Sci. 544:443-450.

2. Powderly, W.G. 1992. Therapy for cryptococcal meningitis in patients with AIDS. Clin. Infect. Dis. 14(Suppl. 1):S54-S59.

3. Diamond, R.D., and J.E. Bennett. 1974. Prognostic factors in cryptococcal meningitis. A study of 111 cases. Ann. Intern. Med. 80:176-180.

4. Farmer, S.G., and R.A. Komoromski. 1973. Histologic response to capsule-deficient Cryptococcus neoformans. Arch. Pathol. 96:383-387.

5. Rippon, J.W. 1988. Cryptococcosis. In Medical Mycology, the Pathogenic Fungi and the Pathogenic Actinomytes. J.W. Rippon, editor. W.B. Saunders Co., Philadelphia. 595-599.

6. Dong, Z.M., and J.W. Murphy. 1995. Intravascular cryptococcal culture filtrate (CneF) and its major component, glucuronoxylomannan, are potent inhibitors of leukocyte accumulation. Infect. Immun. 63:770-778.

7. Butcher, E.C. 1991. Leukocyte-endothelial cell recognition: three (or more) steps to specificity and diversity. Cell. 67:1033-1036.

8. Springer, T.A. 1994. Traffic signals for lymphocyte recirculation and leukocyte emigration: the multistep paradigm. Cell. 76:301-314

9. Jutila, M.A. 1993. Function and regulation of leukocyte homing receptors. J. Leukocyte Biol. 55:133-140.

10. Jutila, M.A., L. Rott, E.L. Berg, and E.L. Butcher. 1989. Function and regulation of the neutrophil Mel-14 antigen in vivo: comparison with LFA-1 and Mac-1. J. Immunol. 143:3318-3324.

11. Granert, C., J. Raud, X. Xie, L. Lindquist, and L. Lindbom. 1994. Inhibition of leukocyte rolling with polysaccharide fucoidin prevents pleocytosis in experimental meningitis in the rabbit. J. Clin. Invest. 93:929-936.

12. Schleiffenbanm, B., O. Spertini, and T.F. Tedder. 1992. Soluble L-selectin is present in human plasma at high levels and retain functional activity. $J$. Cell Biology. 119:229-238.
13. Simonet, W.S., T.M. Hughes, H.Q. Ngugen, L.D. Trebasky, D.M. Danilenko, and E.S. Medlock. 1994. Long-term impaired neutrophil migration in mice overexpressing human interleukin-8. J. Clin. Invest. 94:1310-1319.

14. Murphy, J.W., R.L. Mosley, R. Cherniak, G.H. Reyes, T.R. Kozel, and E. Reiss. 1988. Serological, electrophoretic, and biological properties of Cryptococcus neoformans antigens. Infect. Immun. 56:424-431.

15. Cherniak, R., and J.B. Sundstrom. 1994. Polysaccharide antigens of the capsule of Cryptococcus neoformans. Infect. Immun. 62:1507-1512.

16. Reiss, E., R. Cherniak, E. Eby, and L. Kaufman. 1984. Enzyme immunoassay detection of IgM to galactoxylomannan of Cryptococcus neoformans. Diagn. Immunol. 2:109-115.

17. Buchanan, K.L., and J.W. Murphy. 1993. Characterization of cellular infiltrations and cytokine production during the expression phase of the anticryptococcal delayed-type hypersensitivity response. Infect. Immun. 61:2854-2865.

18. Dong, Z.M., and J.W. Murphy. 1993. Mobility of human neutrophils in response to Cryptococcus neoformans cells, culture filtrate antigen, and individual components of the antigen. Infect. Immun. 61:5067-5077.

19. Tedder, T.F., A.C. Penta, H.B. Levine, and A.S. Freedman. 1990. Expression of the human leukocyte adhesion molecule, LAM1. J. Immunol. 144: $532-540$.

20. Luscinskas, F.W., J.W. Kiely, H. Ding, M.S. Obin, C.A. Hebert, J.B. Baker, and M.A. Gimbrone, Jr. 1992. In vitro inhibitory effect of IL-8 and other chemoattractants on neutrophil-endothelial adhesive interactions. J. Immunol. 149:2163-2171.

21. Bazil, V., and J.L. Strominger. 1994. Metalloprotease and serine protease are involved in cleavage of CD43, CD44, and CD16 from stimulated human granulocytes. J. Immunol. 152:1314-1322.

22. Porteu, F., and C. Nathan. 1990. Shedding of tumor necrosis factor receptor by activated human neutrophils. J. Exp. Med. 172:599-607.

23. Huizinga, T.W., C.E. van der Schoot, C. Jost, R. Klaassen, M. Kleijer, A.E.G.K. von dem Borne, D. Roos, and P.A.T. Tetteroo. 1988. The PI-linked receptor FcRIII is released on stimulation of neutrophils. Nature (Lond.). 333: 667-669.

24. Larsen, E., T. Palabrica, S. Sajer, G.C. Gilbert, D.D. Wagner, B.C. Furie, and B. Furie. 1990. PADGEM-dependent adhesion of platelets to monocytes and neutrophils is mediated by a lineage-specific carbohydrate, LNF III (CD15). Cell. 63:467-474.

25. Brockhaus, M., H.J. Schonfeld, E.J. Schlaeger, W. Hunziker, W. Lesslauer, and H. Loetscher. 1990. Identification of two types of tumor necrosis factor receptors on human cell lines by monoclonal antibodies. Proc. Natl. Acad. Sci. USA. 87:3127-3133.

26. Murphy, J.W. 1989. Cyptococcosis. In Immunology of the Fungal Diseases. R.A. Cox, editor. CRC press, Boca Raton, FL. 97 pp.

27. Eng, R.H.K., E. Bishburg, S.M. Smith, and R. Kapila. 1986. Cryptococcal infections in patients with acquired immune deficiency syndrome. Am. J. Med. 81:19-23.

28. Kozel, T.R., and E.C. Gotschlich. 1982. The capsule of Cryptococcus neoformans passively inhibits phagocytosis of the yeast by macrophages. J. Immunol. 129:1675-1680.

29. Murphy, J.W., and G.C. Cozard. 1972. Immunological unresponsiveness induced by cryptococcal capsular polysaccharide assayed by the hemolytic plaque technique. Infect. Immun. 5:896-901.

30. Murphy, J.W., and J.W. Moorhead. 1982. Regulation of cell-mediated immunity in cryptococcosis. I. Induction of specific afferent T suppressor cells by cryptococcal antigen. J. Immunol. 128:276-282.

31. Collins, H., and G.J. Bancroft. 1991. Encapsulation of Cryptococcus neoformans impairs antigen-specific T-cell responses. Infect. Immun. 59:38833888.

32. Lovchik, J.A., and M.F. Lipscomb. 1993. Role for C5 and neutrophils in the pulmonary intravascular clearance of circulating Cryptococcus neoformans. Am. J. Respir. Cell Mol. Biol. 9:617-627.

33. Miller, M.F., and T.G. Mitchell. 1991. Killing of Cryptococcus neoformans strains by human neutrophils and monocytes. Infect. Immun. 59:24-28.

34. Kozel, T.R., M.A. Wilson, and J.W. Murphy. 1991. Early events in initiation of alternative complement pathway activation by the capsule of Cryptococcus neoformans. Infect. Immun. 59:3101-3110.

35. Fong, Y., and S.F. Lowry. 1990. Tumor necrosis factor in the pathophysiology of infection and sepsis. Clin. Immunol. Immunopathol. 55:157-170.

36. Shalaby, M.R., M.A. Palladino, Jr., S.E. Hirabayashi, T.E. Eessalu, G.D. Lewis, H.M. Shepard, and B.B. Aggarwal. 1987. Receptor binding and activation of polymorphonuclear neutrophils by tumor necrosis factor-alpha. J. Leukocyte Biol. 41:196-204.

37. Lantz, M., F. Bjoruberg, I. Olsson, and J. Richter. 1994. Adherence induces release of soluble tumor necrosis factor receptor forms. J. Immunol. 152: 1362-1369.

38. Stocks, S.C., M. Albrechtsen, and M.A. Kerr. 1990. Expression of the CD15 differentiation antigen (3-fucosyl- $N$-acetyl-lactosamine, $\mathrm{Le}^{\mathrm{X}}$ ) on putative neutrophil adhesion molecules CR3 and NCA-160. Biochem. J. 268:275-285.

39. Carlos, T.M., and J.M. Harlan. 1994. Leukocyte-endothelial adhesion molecules. Blood. 84:2068-2101.

40. Diaz-Gonzalez, F., I. Gonzalez-Alvaro, M.R. Campanero, F. Mollinedo, M.A.D. Pozo, C. Munoz, J.P. Pivel, and F. Sanchez-Madrid. 1995. Prevention of 
in vitro neutrophil-endothelial attachment through shedding of L-selectin by nonsteroidal antiinflammatory drugs. J. Clin. Invest. 95:1756-1765.

41. Hechtman, D.H., M.I. Cybulsky, H.J. Fuchs, J.B. Baker, and M.A. Gimbrone, Jr. 1991. Intravascular IL-8. J. Immunol. 147:883-892.

42. Kishimoto, T.K., M.A. Jutila, E.L. Berg, and E.C. Butcher. 1989. Neutrophil Mac-1 and Mel-14 adhesion proteins are inversely regulated by chemotactic factors. Science (Wash. DC). 245:1238-1241.

43. Enders, G., W. Brooks, N.V. Jan, N. Lehn, E. Bayerdorffer, and R.
Hatz. 1995. Expression of adhesion molecules on human granulocytes after stimulation with Helicobacter pylori membrane proteins: comparison with membrane proteins from other bacteria. Infect. Immun. 63:2473-2477.

44. Palecanda, A., B. Walcheck, D.K. Bishop, and M.A. Jutila. 1992. Rapid activation-independent shedding of leukocyte L-selectin induced by cross-linking of the surface antigen. Eur. J. Immunol. 22:1279-1286.

45. Weston, S.A., and C.R. Parish. 1991. Modification of lymphocyte migration by mannans and phosphomannans. J. Immunol. 146:4180-4186. 